\title{
Focus on surgical care of the adolescent trauma patient
}

\author{
D. W. Sommerfeldt
}

Received: 15 October 2013/Accepted: 11 November 2013/Published online: 3 December 2013

(C) Springer-Verlag Berlin Heidelberg 2013

Adolescents are "special." In many ways. Every parent who has or had one at home for a while knows this for a fact. This also holds true for trauma care within this specific age group. Why? Because in several categories, including the etiology of injuries, the psychology of the patient pre-, intra-, and postoperatively, skeletal biology, and the rehabilitation process, specific considerations have to be taken into account to achieve optimum results.

Etiology Risk-taking is one of the main personality traits of teenagers and adolescents. They prefer high velocities in ground transportation and sports. Within the group of trauma patients aged from 0 to 18 years, the age group of 14-17 years comprises more than two-thirds of the trauma load. In addition, sports injuries tend to be more serious within this age group when compared to younger athletes.

Psychology The need to explore their limits can lead to serious injuries within this age group, but the adolescent can also be a difficult patient to treat once surgery becomes necessary. Parental consent is still mandatory, of course, but the teenager him/herself also needs to be well informed about the seriousness and the consequences of his/her injury in order to be able to cooperate during treatment postoperatively and during the rehabilitation process. This is not always easy, and problems with anger management and frustration can occur during the healing process.

Biology Musculoskeletal biology is different from children and from adults at this age. Growth plates may still be wide open or can be close to fusion. If they are still open,

D. W. Sommerfeldt $(\bowtie)$

Pediatric and Adolescent Orthopaedic Trauma, German Society of Traumatology, Bleickenallee 38, 22763 Hamburg, Germany

e-mail: dirk.sommerfeldt@kinderkrankenhaus.net posttraumatic growth disturbances, including leg length discrepancies and axial deformity, can become a problem for the patient and the treating physician. If the growth plates are close to fusion, they become biomechanically unstable for a period of several months, which can lead to characteristic injuries such as epiphysiolysis and apophysitis. In the adolescent athlete, specific cartilaginous injuries can occur within the joints, such as fracture of the medial epicondyle following a dislocated elbow. However, chronic cartilage problems such as osteochondrosis dissecans of the elbow, knee, or ankle or pitcher's elbow (chronic apophysitis of the medial epicondyle of the humerus) can be mistaken for an acute injury, leading to wrong therapeutic consequences by the physician.

Rehabilitation In children, physical therapy is rarely necessary following skeletal injuries, even when a fracture has been treated conservatively with a cast for several weeks. Free range of movement is usually achieved within a few weeks, at the latest within 2 months, and the natural wish of young children to move and run around is largely responsible for this phenomenon.

Within the adolescent age group, however, the rehabilitation process can be prolonged. Again, this may have psychological reasons, but also the ligaments, muscles, and bones of the adolescent patient behave much more like adult ones, so they may need a specific physiotherapy regimen that takes into account both the psychological and biological specifics of these patients.

In summary, adolescents are special patients. Transition medicine is starting to pick up on this, and the orthopedic and trauma surgeon needs to be aware of these specifics when treating injuries in the adolescent patient. Therefore, we have invited trauma experts to elaborate on the characteristic injuries and problems they have encountered 
within this age group. The topics cover a wide range, from long bone fractures to joint injuries, and we hope that the information given will create awareness of the specific requirements from the perspective of the trauma surgeon, and will make treating these young patients easier and even more rewarding for the reader. Because nothing is more fun than accompanying a patient through surgery and rehabilitation, and having him or her perform at a high level again, be it in sports or within the activities of daily life.

Conflict of interest None. 